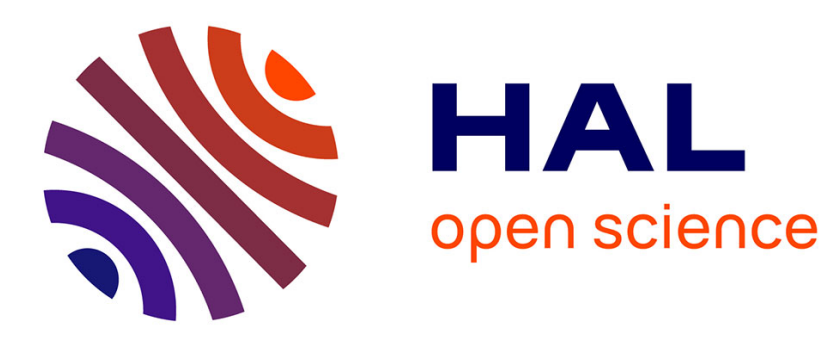

\title{
Discrimination of ordinal relationships in temporal sequences by 4-month-old infants
}

Maria Dolores de Hevia, Viola Macchi Cassia, Ludovica Veggiotti, Maria Eirini Netskou

\section{- To cite this version:}

Maria Dolores de Hevia, Viola Macchi Cassia, Ludovica Veggiotti, Maria Eirini Netskou. Discrimination of ordinal relationships in temporal sequences by 4-month-old infants. Cognition, 2020, 195, pp.104091. 10.1016/j.cognition.2019.104091 . hal-02372264

\section{HAL Id: hal-02372264 \\ https://hal.science/hal-02372264}

Submitted on 21 Dec 2021

HAL is a multi-disciplinary open access archive for the deposit and dissemination of scientific research documents, whether they are published or not. The documents may come from teaching and research institutions in France or abroad, or from public or private research centers.
L'archive ouverte pluridisciplinaire HAL, est destinée au dépôt et à la diffusion de documents scientifiques de niveau recherche, publiés ou non, émanant des établissements d'enseignement et de recherche français ou étrangers, des laboratoires publics ou privés.

\section{(c)(1) $\$$}

Distributed under a Creative Commons Attribution - NonCommerciall 4.0 International 


\title{
Discrimination of ordinal relationships in temporal sequences by 4-month-old infants
}

\author{
Maria Dolores de Hevia ${ }^{1,2}$, Viola Macchi Cassia ${ }^{3,4}$, Ludovica Veggiotti ${ }^{1}$, \& Maria Eirini \\ Netskou $^{1}$
}

1. Université Paris Descartes, Sorbonne Paris Cité, Paris, France

2. CNRS UMR 8002, Integrative Neuroscience and Cognition Center, Paris, France

3. Department of Psychology, University of Milano-Bicocca, Milan, Italy

4. NeuroMi, Milan Center for Neuroscience, Milan, Italy

Running Head: Ordinal discrimination for temporal sequences 
1 Discrimination of ordinal relationships in temporal sequences by 4-month-old 2 infants

3

4

5

6 Running Head: Ordinal discrimination for temporal sequences

7

8

9

10

11

12

13 


\section{Abstract}

The ability to discriminate the ordinal information embedded in magnitude-based sequences has been shown in 4-month-old infants, both for numerical and size-based sequences. At this early age, however, this ability is confined to increasing sequences, with infants failing to extract and represent decreasing order. Here we investigate whether the ability to represent order extends to duration-based sequences in 4-month-old infants, and whether it also shows the asymmetry signature previously observed for number and size. Infants were tested in an order discrimination task in which they were habituated to either increasing or decreasing variations in temporal duration, and were then tested with novel sequences composed of new temporal items whose durations varied following the familiar and the novel orders in alternation. Across three experiments, we manipulated the duration of the single temporal items and therefore of the whole sequences, which resulted in imposing more or less constraints on infants' working memory, or general processing capacities. Results showed that infants failed at discriminating the ordinal direction in temporal sequences when the sequences had an overall long duration (Experiment 1), but succeeded when the duration of the sequences was shortened (Experiments 2 and 3). Moreover, there was no sign of the asymmetry signature previously reported for number and size, as successful discrimination was present for infants habituated to both increasing and decreasing sequences. These results suggest that sensitivity to temporal order is present very early in development, and that its functional properties are not shared with other magnitude dimensions, such as size and number.

Keywords: ordering operations, temporal duration, habituation, infants 


\section{Introduction}

Temporal duration is one of the basic quantitative attributes of the environment that, together with numerical quantity and space, is fundamental in our cognitive lives (e.g., Meck \& Church, 1983; Walsh, 2003). In humans, the ability to time events (e.g., the duration of a tone, or the duration of a visual object coupled with a sound) develops very early in life and is functional at birth (see de Hevia, Lee, \& Streri, 2017 for a review). Infants in their first year of life are able to discriminate between the different temporal durations of two audiovisual events created by a puppet coupled with a tone. For instance, 6-month-old infants that have been habituated to an event duration of $2 \mathrm{~s}$ and are then tested with the same event lasting for $2 \mathrm{~s}$ in some trials or $4 \mathrm{~s}$ in other trials, show increased attention to the trials containing the novel event duration (Brannon, Suanda, \& Libertus, 2007; vanMarle \& Wynn, 2006).

Critically, the precision with which temporal events are encoded improves with age. Sixmonth-old infants need at least a 1:2 ratio difference (e.g., $2 \mathrm{~s}$ vs. $4 \mathrm{~s}$, or $3 \mathrm{~s}$ vs. $6 \mathrm{~s}$ ) between durations for a successful discrimination, as they fail at discriminating two events whose durations differ by a 2:3 ratio (e.g., 3 s vs. 4.5 s, or .67 s vs. 1 s) (vanMarle $\&$ Wynn, 2006). But successful discrimination occurs with this same ratio $2: 3$ (i.e., $1 \mathrm{~s}$ vs. $1.5 \mathrm{~s}$ and $2 \mathrm{~s}$ vs. $3 \mathrm{~s}$ ) at 10 months of age, when, however, infants fail at discriminating durations that differ by a 3:4 ratio (i.e., $3 \mathrm{~s}$ vs. $4 \mathrm{~s}$ ), suggesting that this smaller ratio is approaching the upper limit of their capacity (Brannon et al., 2007).

The main cognitive attribute characterizing representations of quantitative dimensions, such as duration, is their analog format (Gallistel \& Gelman, 2000). In fact, the ability to time events and to discriminate between event durations is determined by Weber's law, whereby the discriminability between two magnitudes depends on the ratio, not the absolute difference, 
between their values (Meck, 2003). This signature of quantity processing has been shown to apply to a variety of perceptual dimensions in adults, from number (Van Oeffelen \& Vos, 1982), to spatial extent (Johnson, 1939), and other dimensions such as brightness and loudness (Stevens

\& Marks, 1965). The developmental trajectory of temporal discrimination parallels the ones described for discrimination of numerical information and spatial extent in preverbal infants: at six months of age, infants need a 1:2 ratio to discriminate between two different numerosities (Lipton \& Spelke, 2003; Xu \& Spelke, 2000), or between the size of two objects (Brannon, Lutz, $\&$ Cordes, 2006), while the ratio can be lowered to 2:3 at nine months of age (Brannon et al., 2006; Lipton \& Spelke, 2003). The equivalent developmental trajectories in the representation of number, area or spatial extent, and time, support the idea that magnitude representations may be rooted in a single developmental algorithm for 'more than/less than' distinctions (for reviews, see Bonn \& Cantlon, 2012; de Hevia, 2016a; Feigenson, 2007). Another central aspect of the representation of quantitative dimensions is their ordinal property, by which distinct instances of a given magnitude dimension can be put in a 'greater than' or 'less than' relation to one another. In the domain of number, a series of studies have shown that the ability to represent ordinal information among different (i.e., at least three) numerosities is functional during the first year of life. Picozzi and colleagues (Picozzi, de Hevia, Girelli, \& Macchi Cassia, 2010) found that 7-month-old infants are able to detect a reversal in the ordinal direction (i.e., increasing vs. decreasing order) of numerical sequences, and do so when the corresponding variations in other non-numerical quantities such as surface area, density, and contour length are carefully controlled. After habituation to three-item numerical sequences changing progressively by a 1:2 ratio, infants looked longer at numerical sequences displaying the reversed ordinal direction: infants habituated to sequences of 6,12 , and 24 objects 
looked longer to a sequence of 16,8 , and 4 objects than to a sequence of 4,8 , and 16 at test, and this preference reversed in infants habituated to sequences of 24,12 , and 6 objects. Similarly, for the dimensions of size and time, studies with 8- to 9-month-old infants have shown that they are able to detect the reversal of order in sequences where the size/duration of objects progressively increases or decreases (de Hevia \& Spelke, 2010; Srinivasan \& Carey, 2010).

Critically, it has been recently shown that the ability to discriminate numerical and size order follows a developmental course over the first year of life. Although by 7-8 months infants are able to process and discriminate increasing vs. decreasing numerical sequences (de Hevia \& Spelke, 2010; Picozzi et al., 2010) as well as size and temporal sequences (Brannon, 2002; de Hevia \& Spelke, 2010; Srinivasan \& Carey, 2010), infants tested at 4 months of age showed evidence of discrimination only following habituation to increasing, but not decreasing sequences, both for number (de Hevia, Addabbo, Nava, Croci, Girelli, \& Macchi Cassia, 2017) and size (Macchi Cassia, Picozzi, Girelli, \& de Hevia, 2012). Importantly, infants were shown to treat equally decreasing sequences of items varying in physical size and random (unordered) sequences (Macchi Cassia et al., 2012, Exp. 1b). It was therefore concluded from these studies that younger infants might represent the decreasing series as a collection of unordered instances of magnitude, with no encoding of the directionality of the magnitude change (see extensive discussions in de Hevia et al., 2017 and Macchi Cassia et al., 2012). This asymmetry signature in ordinal processing (i.e., success for increasing order and failure for decreasing order) for number and size was tentatively interpreted as emerging from an evolutionary mechanism related to perceptual 'looming' (i.e., the ability to react defensively to the perceived impending collision of an approaching stimulus, which is functional shortly after birth, Náñez, 1988), since detecting and keeping track of the increasing magnitude embedded in approaching stimuli might have been 
selected as being potentially critical for survival. Moreover, it has been proposed that the asymmetry signature might be at the roots of related cognitive operations that carry in their performance pattern this computational attribute. In particular, the authors proposed that the asymmetry might constitute a developmental precursor of the 'addition advantage' (i.e., better performance and earlier acquisition of addition relative to subtraction arithmetic operations), which permeates mathematical performance from childhood to adulthood (e.g., Barth, Beckmann, \& Spelke, 2008; Campbell \& Xu, 2001). The evidence for a common (or at least parallel) development of ordinal representation for number and size leaves open the question of whether the signature of asymmetric sensitivity to order in 4-month-old infants, with successful detection of increasing order coupled with failure at discriminating decreasing order, extends to other magnitude dimensions.

Building on this earlier evidence, in the current study we tested 4-month-old infants' ability to detect a reversal in the ordinal direction of duration-based sequences. If, as some theories suggest, different quantity dimensions (particularly number, space and time) are represented via a common representational code (ATOM theory: Bueti \& Walsh, 2009; Walsh, 2004), we would expect that the same processing constraints apply to these dimensions. This prediction is already partially supported by the finding that the same ratios are required across development to successfully discriminate unequal values for the dimensions of number, size, and time (Brannon et al., 2006; Feigenson, 2007), and that human newborns and infants spontaneously relate quantities across these dimensions and expect them to vary in congruent ways (de Hevia, Izard, Coubart, Spelke, \& Streri, 2014; de Hevia \& Spelke, 2010; Srinivasan \& Carey, 2010). More crucially, the hypothesis that the same computational mechanisms and constraints characterize the processing of number and size is supported by the finding of a 
common asymmetry signature in ordinal processing for both of size-based (Macchi Cassia et al., 2012) and numerical sequences (de Hevia et al., 2017). Therefore, we should expect that the same asymmetry in ordinal processing would extend to the dimension of time.

However, both adult and developmental literature also offer counter evidence to the hypothesis of shared processing signatures for all magnitude dimensions. In fact, a number of findings point to the existence of important differences in functional properties and developmental trajectories for the representations of number, space (or size) and time. While in early stages of development the precision with which infants represent instances of number, size and time is the same, developmental trajectories part ways during childhood, with precision in time representation not fully developing until early adulthood, space (area and length) representation developing by adolescence, and number representation developing at an intermediate rate (Odic, 2018). Recent research with adults has also reported discrepancies in the processing of these dimensions, with higher precision for numerical than for temporal judgments (Odic, Lisboa, Eisinger, Olivera, Maiche, \& Halberda, 2016), and cognitive load and emotional valence of the stimuli differentially affecting number and time processing within the same participants (Hamamouche, Keefe, Jordan, \& Cordes, 2018; Young \& Cordes, 2013). Of note, recent studies have shown that some differences in the processing of number, space and time are already in place in the earliest stages of human development, as newborn infants associate small numerosities to the left and large numerosities to the right side of space, but the same spatial association is not present for the dimensions of size and time (de Hevia, Veggiotti, Streri, \& Bonn, 2017; see also Bulf, de Hevia, \& Macchi Cassia, 2016 for older infants).

Therefore, with the literature offering a general picture of both parallelisms and divergences in the representation of the quantitative dimensions of number, space and time, it is 
an open question whether the processing of ordinal relationships among magnitudes across these

2 dimensions presents common signatures in early infancy. In the present study we explored this

3 question in three experiments where 4-month-old infants were tasked with ordinal discrimination

4 for duration-based sequences.

\section{Experiment 1}

In Experiment 1, 4-month-old infants were habituated with a series of increasing or

8 decreasing duration-based sequences and were then tested with new sequences in which the same

9 ordinal direction and the reverse ordinal direction were presented in alternation. If infants are able to represent order in the temporal dimension, they should look longer during test at the sequences exhibiting the new ordinal direction with respect to the one they have been habituated to (i.e., decreasing for those habituated to increasing sequences and increasing for those habituated to decreasing sequences).

\section{Methods}

Methods were modelled after de Hevia et al. (2017) and Macchi Cassia et al. (2012), where 4-month-old infants were tested in their abilities to represent ordinal information for numerical and size-based sequences, respectively.

\section{Participants}

Participants were 24 healthy, full-term 4-month-old infants (11 females, mean age $=4$ months 3 days, range $=3$ months, 14 days -4 months, 22 days). Data from an additional 17 infants were excluded from the final sample because they failed to complete testing due to 
1 fussiness or lack of interest $(n=10)$, looking time in at least one test trial more than 3 standard

2 deviations (SD) from the overall group mean $(n=4)$, experimental error $(n=1)$, or parental

3 interference $(\mathrm{n}=2)$.

4

\section{Ethics Statement}

The methods used in the study received approval from the Ethics Committee of

University Paris Descartes. Parents gave their written informed consent before testing began.

8

9

Stimuli

Stimuli were adapted from Macchi Cassia et al. (2012, Study 2); they were single colored shapes of fixed area $\left(24 \mathrm{~cm}^{2}\right)$ appearing for variable durations, either in increasing or decreasing order, changing by a 1:2 ratio (range 1-7.2 s), and accompanied by a tone of a corresponding duration (amplitude $=0.8$; frequencies $=440,450,460,470 \mathrm{~Hz}$ ). Stimuli were presented against a white background.

There were four sets of stimuli: three for the habituation phase and one for the test phase, each set being composed of a different shape and color, and accompanied by a unique frequency tone. The three habituation sets contained green triangles (accompanied by a $450 \mathrm{~Hz}$ tone with the corresponding duration), red squares ( $460 \mathrm{~Hz}$ tone), and blue circles (440 Hz tone), with a duration of, respectively, 1, 2, $4 \mathrm{~s} ; 1.4,2.8,5.6 \mathrm{~s}$; and 1.8, 3.6, 7.2 s for the increasing order, and 4, 2, $1 \mathrm{~s} ; 5.6,2.8,1.4 \mathrm{~s}$; and 7.2, 3.6, $1.8 \mathrm{~s}$ for the decreasing order. The test set contained purple rectangular figures with a duration of $1.5,3,6 \mathrm{~s}$ for increasing order and $6,3,1.5 \mathrm{~s}$ for decreasing order, accompanied by a $470 \mathrm{~Hz}$ tone with the corresponding duration. Therefore, within each sequence an item differed from the previous one only in duration, following a 1:2 ratio. 


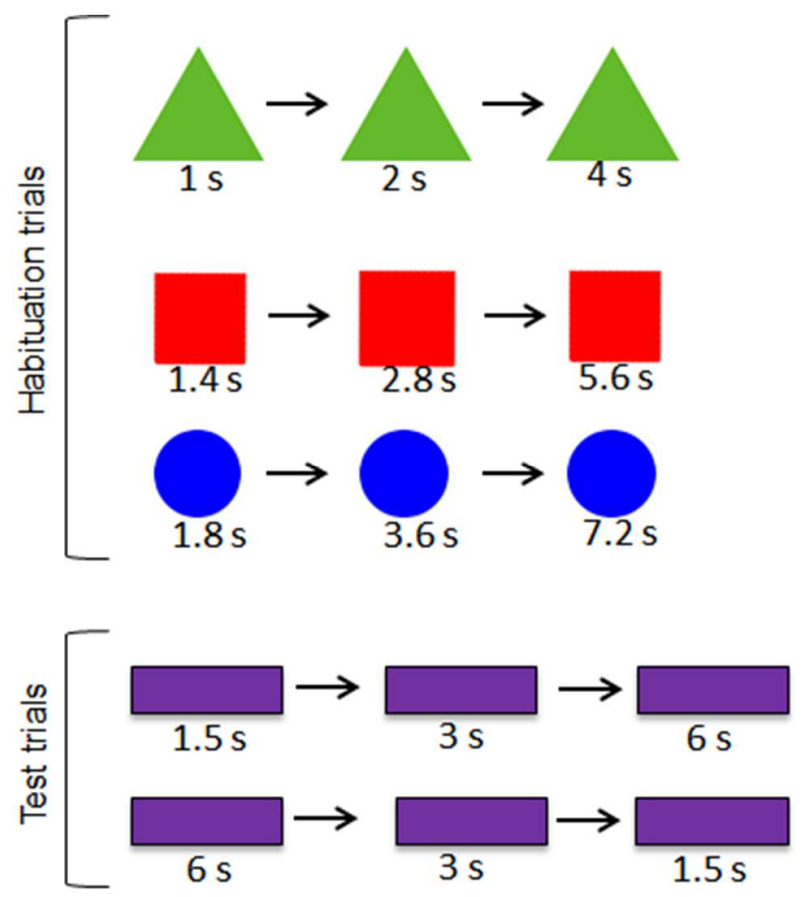

2 Figure 1. The five sets (three for habituation, two for test) of colored shapes, and their duration (seconds), as they

3 were presented to infants in the increasing order condition of Experiment 1. Arrows represent passage of time;

4 objects were presented serially, centered on the screen. Green triangles, red squares and blue circles were presented

5 in a fixed order during habituation, and the purple rectangles were presented during test, exhibiting both the familiar

6 and the novel duration-based orders in alternation, with presentation order counterbalanced across participants. For

7 infants in the decreasing order condition the same habituation shapes were presented in reversed order (from the

8 longer to the shorter durations), starting from the blue circles, and proceeding to the red squares and green triangles,

9 and the test trials were the same as in the increasing habituation conditions, alternating familiar and novel duration-

10 based orders, with order presentation counterbalanced across participants.

During habituation, half of the infants were exposed to increasing temporal sequences and the other half to decreasing sequences. Infants were randomly assigned to each habituation condition. Within each habituation condition, the three different stimulus sets were cycled in a

16 fixed order until infants met the habituation criterion: from the shortest to the longest duration 
1 for the increasing condition (i.e. $1,2,4 \mathrm{~s} ; 1.4,2.8,5.6 \mathrm{~s} ; 1.8,3.6,7.2 \mathrm{~s}$ ), and from the longest to

2 the shortest duration for the decreasing condition (i.e. 7.2, 3.6, $1.8 \mathrm{~s} ; 5.6,2.8,1.4 \mathrm{~s} ; 4,2,1 \mathrm{~s}$ ).

3 The use of a consistent fixed order of presentation of the sets across trials for each of the two

4 habituation conditions provided infants with redundant cues to ordinality between, as well as

5 within, trials (see de Hevia et al., 2017; Macchi Cassia et al., 2012; Picozzi et al., 2010).

6 Following habituation, all infants were given six test trials alternating the familiar and the novel

7 orders in sequences of purple rectangles (i.e., 1.5, 3, $6 \mathrm{~s} ; 6,3,1.5 \mathrm{~s}$ ), with the order of

8 presentation counterbalanced across participants. For infants in the increasing habituation

9 condition the familiar test sequence comprised purple rectangles presented for $1.5,3,6 \mathrm{~s}$, and the 10 novel test sequence comprised purple rectangles presented for $6,3,1.5 \mathrm{~s}$; the opposite was true 11 for infants in the decreasing habituation condition.

Apparatus

Each infant was tested while sitting in an infant seat approximately $60 \mathrm{~cm}$ from the monitor where the stimuli were presented (23" screen size, 1920 X 1080 pixel resolution, refresh rate of $60 \mathrm{~Hz}$ ) in a dimly lit room. A video camera was positioned just above the stimulus presentation monitor and directed to the infant's face. The live image of the infant's face was displayed on a television monitor to allow the online coding of the infant's looking times through the E-Prime 1.0 program by the experimenter, who was blind to the habituation condition to which the infant was assigned and the order of test trials. The image of the infant's face was also recorded via a Mini-DV digital recorder, and data from all test trials were subsequently coded offline by a second coder. Intercoder agreement (Pearson correlation) between the two observers who coded the data live or from digital recording, as computed on total fixation times on each of 
1 the six test trials, was $r=.967$. For each test trial, the average between the data coded live and

2 offline was computed and employed as the dependent variable (if data from two coders differed

3 by more than $5 \mathrm{~s}$, a third coder was used, and the average between the two nearest values was

4 taken).

$5 \quad$ Procedure

Each trial began as soon as the infant looked in the direction of an attention getter (a

7 cartoon-animated image associated to a varying sound) displayed in the center of the screen. The

8 trial started by showing a dark gray screen $(500 \mathrm{~ms})$, followed by a light gray screen $(250 \mathrm{~ms})$

9 appearing before each of the three stimuli composing the sequence. The Inter-Stimulus Interval (ISI) between the sequences was $500 \mathrm{~ms}$. Each trial continued until the infant looked away continuously for $2 \mathrm{~s}$ or looked for a maximum of $130 \mathrm{~s}$. Looks away shorter than $2 \mathrm{~s}$ did not stop the trial, and for a single look to be considered valid it had to have a minimum duration of 500 ms. The three habituation stimulus sets were presented in a fixed order and repeated until the infant saw a maximum of 14 trials or met the habituation criterion, which was defined as a $50 \%$ decline in looking time on three consecutive trials, relative to the looking time on the first three trials. Following the habituation phase, infants were given six test trials, alternating between the familiar and the novel orders, with half of the infants seeing the familiar order first.

\section{$\underline{\text { Results and Discussion }}$}

All statistics are two-tailed. The average number of trials received during habituation did not differ for infants tested in the increasing and decreasing order conditions ( $M=9.33$ trials vs. $M=7.83$ trials, $t(22)=1.09, p=.29$, independent-samples t-test). A two-way Analysis of Variance (ANOVA) on mean habituation looking times with order condition (increasing vs. 
1 decreasing) as the between-participants factor and habituation trials (first three vs. last three) as

2 the within-participants factor confirmed the presence of a significant overall decrease from the

3 first three $(M=32.16 \mathrm{~s}, S E M=4.01)$ to the last three habituation trials $(M=10.4 \mathrm{~s}, S E M=1.29)$,

$4 F(1,22)=31.34, M S e=5680.45, p<.001, \eta_{p}{ }^{2}=.59$, with no main effect or interaction involving

5 the factor order condition $(p s>.25)$ (Figure 2).

6

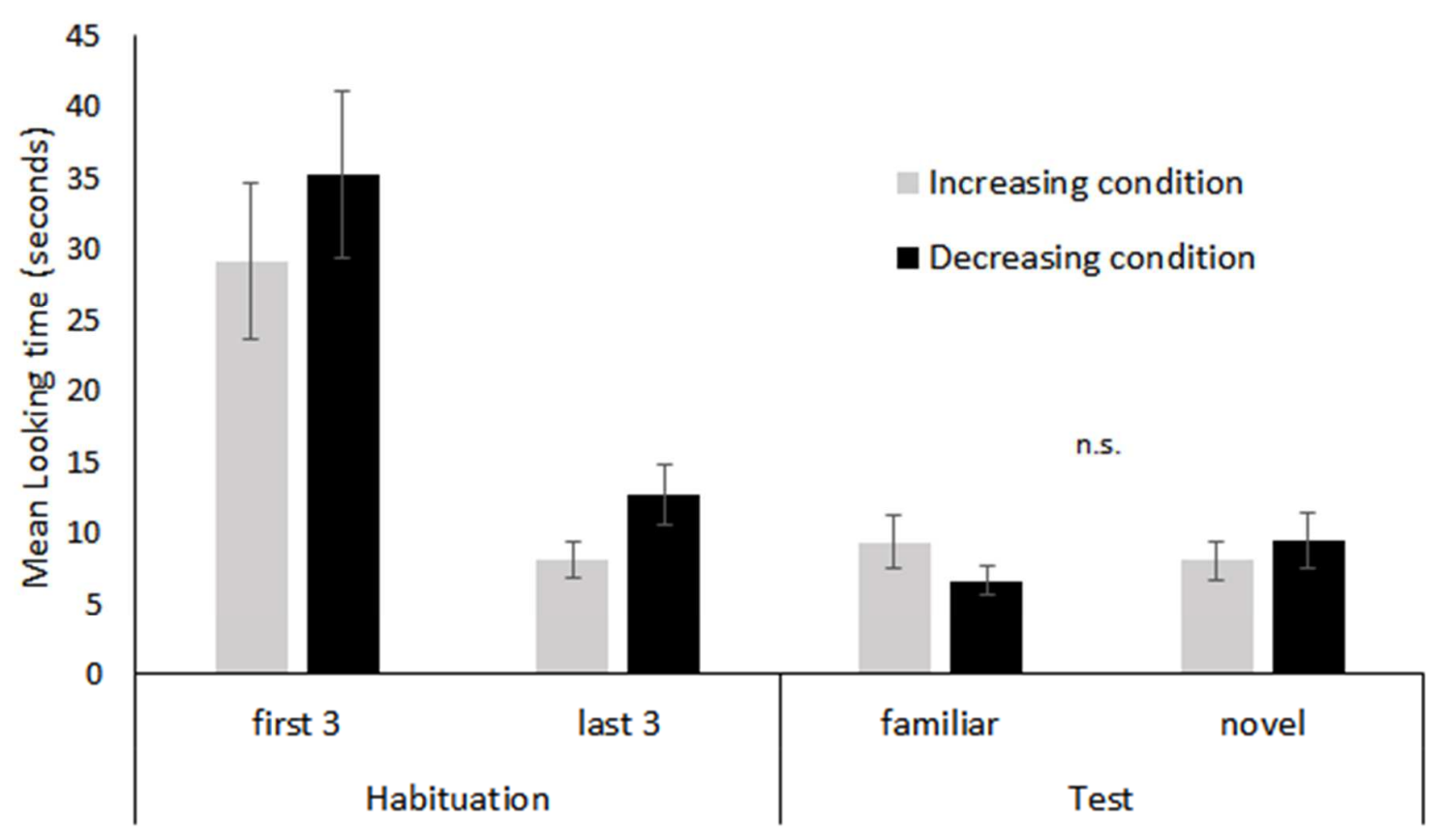

7 Figure 2. Overall mean total looking time $( \pm$ SEM) to the first three and last three habituation trials, and to the

8 familiar and novel test trials displayed by infants in the increasing and decreasing order conditions in Experiment 1.

9 Infants failed to show discrimination between the familiar and the novel ordered sequences at test (n.s., $p=.4)$.

10

11 To determine whether during test trials infants looked longer to the sequences displaying reversed order with respect to the one received during habituation, mean looking times to the test trials were entered into a four-way ANOVA with order condition (increasing vs. decreasing) and test trial order (familiar first vs. novel first) as between-participants factors, and test trial pair 
1 (first vs. second vs. third) and test trial type (familiar vs. novel) as within-participants factors.

2 The analysis revealed a significant main effect of test trial pair, $F(2,40)=18.78, M S e=512.56, p$

$3<.001, \eta_{p}{ }^{2}=.48$, with a progressive decrement of looking time across trial pairs (first pair: $M=$

$411.91 \mathrm{~s}, S E M=2.6$; second pair: $M=7.75 \mathrm{~s}, S E M=1.28$; third pair: $M=5.47 \mathrm{~s}, S E M=.86$; first

5 pair vs. second pair and first pair vs. third pair, both $p s<.01$; second vs. third, $p=.09$, Tukey's

6 HSD post hoc tests). The effect of test trial type was not significant, $F(1,20)<1, M S e=21.01, p$

$7=.38, \eta_{p}{ }^{2}=.038$, but the interactions between test trial type and habituation order condition,

$8 F(1,20)=5.82, M S e=152.93, p=.026, \eta_{p}{ }^{2}=.23$, and between test trial type and test trial order,

$9 F(1,20)=10.6, M S e=278.42, p<.004, \eta_{p}{ }^{2}=.35$, were both significant. We explored these

10 significant interactions through Tukey's HSD post hoc tests, which showed that no comparison

11 attained statistical significance for the Test trial type $\mathrm{x}$ Habituation order condition interaction

12 (increasing habituation condition: novel, $M=8.07 \mathrm{~s}, S E M=2.79$ vs. familiar, $M=9.36, S E M=$

$132.6, p=.70$; decreasing habituation condition: novel, $M=9.45 \mathrm{~s}, S E M=2.79$ vs. familiar, $M=$

$146.63 \mathrm{~s}, S E M=2.6, p=.12$; all other comparisons, $p \mathrm{~s} \geq .56$ ). For the Test trial type $\mathrm{x}$ Test trial

15 order interaction, post hoc tests showed that infants looked significantly longer to the novel than

16 to the familiar order when the first test trial they received included the novel order (novel: $M=$

$1710.77 \mathrm{~s}, S E M=2.79$ vs. familiar: $M=7.23 \mathrm{~s}, S E M=2.6 ; p=.04)$, but not when the first test trial

18 included the familiar order (novel: $M=6.74$ s, $S E M=2.78$ vs. familiar: $M=8.76$ s, $S E M=2.6 ; p$

$19=.4)$. All other effects or interactions were not significant (all $F \mathrm{~s}<4.3, p \mathrm{~s}>.05$ ).

Finally, in order to further explore the Test trial type x Habituation order condition

21 interaction, we analyzed data for individual infants for the two habituation conditions separately

22 using non-parametric tests. In neither of the two order conditions a significant number of infants

23 succeeded to discriminate the novel from the familiar order at test. In the increasing habituation 
condition, only 6 out of 12 infants looked longer to the novel test order ( $p=1$, binomial test); similarly, in the decreasing habituation condition only 8 out of 12 infants looked longer to the novel test order $(p=.388$, binomial test).

Overall, unlike earlier demonstrations of 4-month-olds' successful discrimination of order inversion for numerical (de Hevia et al., 2017) and size-based sequences (Macchi Cassia et al., 2012), our results show that infants did not reliably discriminate the familiar duration-based ordinal sequence from the novel one presented at test. One possible reason for this failure might reside in the fact that, contrary to numerical or size-based visual sequences where each item has the same short duration, in duration-based sequences each item has a different duration and the whole sequence is deployed temporally, therefore imposing a greater cognitive processing load, specifically on working memory capacities. Based on this hypothesis, in Experiments 2 and 3 we further explored 4-month-old infants' ability to represent and discriminate temporal order by progressively diminishing the actual durations of the stimuli and, therefore, the working memory load.

\section{Experiment 2}

In Experiment 2, a new group of 4-month-old infants was habituated with a series of either increasing or decreasing temporal sequences and were then tested with new sequences in which the same ordinal direction and the reversed ordinal direction were presented in alternation. In Experiment 2 we aimed at simplifying the task by shortening the temporal durations of individual audiovisual items (i.e., shapes and tones), and therefore of the overall sequences, so as to impose less constraints on infants' limited working memory capacity. As in Experiment 1, if infants were able to represent the ordinal information embedded in the sequences of different 
1 durations, they should look longer during test at the sequences exhibiting the new ordinal

2 direction with respect to the one they have been habituated to (i.e., decreasing for those

3 habituated to increasing sequences and increasing for those habituated to decreasing sequences).

4

$5 \quad$ Methods

6 The methods were the same as in Experiment 1 except as follows.

$7 \quad$ Participants

The final sample included 24 4-month-olds ( 8 females, mean age $=4$ months 5 days,

$9 \quad$ range $=3$ months 15 days -4 months 14 days). Data from an additional 10 infants were

10 discarded due to fussiness or lack of interest resulting in failure to complete all testing trials $(\mathrm{n}=$

$112)$, or looking time in at least one test trial more than 3SD from the overall group mean $(\mathrm{n}=8)$.

12

13

14

15

16

17

18

19

20

21

22

Stimuli

The habituation and test sets were identical to Experiment 1, except for the duration of presentation of the geometrical figures as well as the associated tone. In particular, the first stimulus set, made of green triangles, had a duration of $0.5,1,2 \mathrm{~s}$ for the increasing habituation condition, and 2, 1, $0.5 \mathrm{~s}$ for the decreasing habituation condition; the red squares had a duration of $0.7,1.4,2.8 \mathrm{~s}$ for the increasing habituation condition, and $2.8,1.4,0.7 \mathrm{~s}$ for the decreasing habituation condition; the blue circles had a duration of $0.9,1.8,3.6 \mathrm{~s}$ for the increasing habituation condition, and 3.6, 1.8, $0.9 \mathrm{~s}$ for the decreasing habituation condition. The set of stimuli presented during test, made of purple rectangles, had a duration of $0.65,1.3,2.6 \mathrm{~s}$ for the increasing sequence and 2.6, 1.3, $0.65 \mathrm{~s}$ for the decreasing sequence. 
A
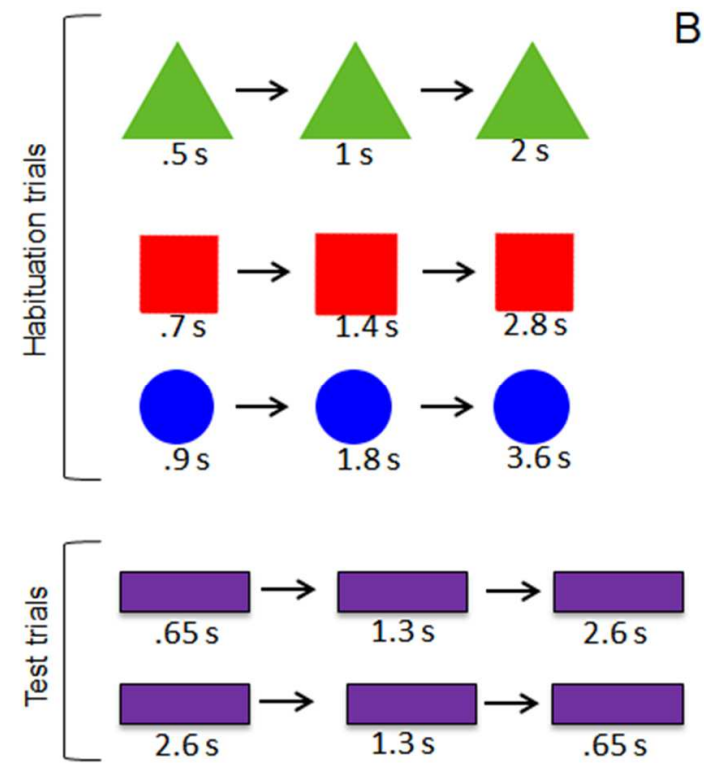

B
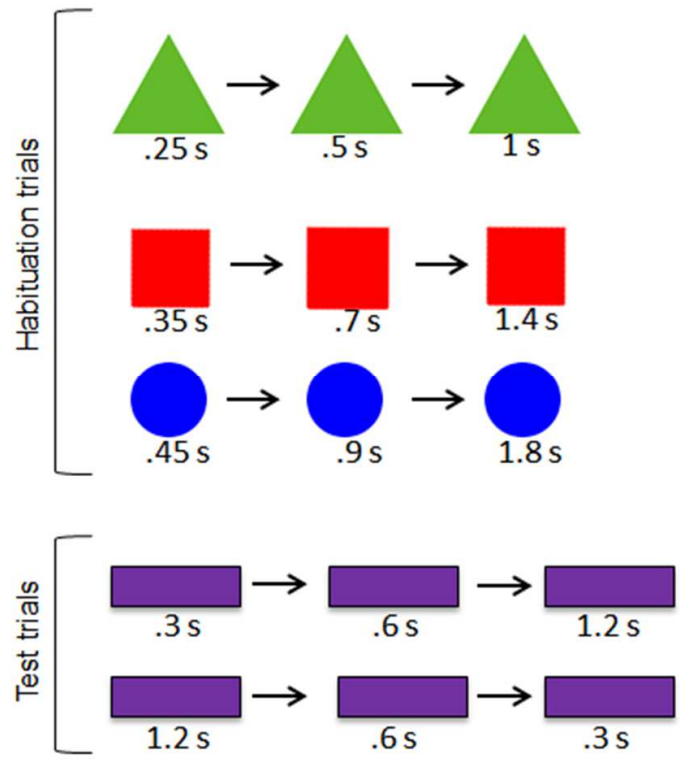

Figure 3. A. The five sets of numerical displays as they were presented to infants in the increasing order condition of Experiment 2. Arrows represent passage of time; objects were presented serially, centered on the screen. The duration-based sequences $0.5,1,2 \mathrm{~s} ; 0.7,1.4,2.8 \mathrm{~s}$; and $0.9,1.8,3.6 \mathrm{~s}$ were presented in a fixed order during habituation, and the duration-based sequences $0.65,1.3,2.6 \mathrm{~s}$ and 2.6, 1.3, $0.65 \mathrm{~s}$ were presented, respectively, during the familiar and the novel test trials, with presentation order counterbalanced across participants. For infants in the decreasing order condition the same habituation displays were presented in reversed order (from the longest to the shortest), starting from the 3.6, 1.8, $0.9 \mathrm{~s}$ sequence and proceeding to the $2.8,1.4,0.7 \mathrm{~s}$, and the $2,1,0.5 \mathrm{~s}$ sequence, and the $2.6,1.3,0.65 \mathrm{~s}$ and the $0.65,1.3,2.6 \mathrm{~s}$ sequences were presented, respectively, during the familiar and the novel test trial. $\underline{B}$. The five sets of numerical displays as they were presented to infants in the increasing order condition of Experiment 3. All being identical to Experiments 1 and 2, only the stimuli durations were changed to: $0.25,0.5,1 \mathrm{~s} ; 0.35,0.7,1.4 \mathrm{~s} ; 0.45,0.9,1.8 \mathrm{~s}$ for the increasing habituation condition; and 1.8, 0.9, $0.45 \mathrm{~s} ; 1.4$, $0.7,0.35 \mathrm{~s} ; 1,0.5,0.25 \mathrm{~s}$ for the decreasing habituation condition; and $0.3,0.6,1.2 \mathrm{~s}$ and $1.2,0.6,0.3 \mathrm{~s}$ for the increasing and decreasing test sequences, respectively. 
$\underline{\text { Results and Discussion }}$

All statistics are two-tailed. The average number of trials received during habituation did not differ for infants in the increasing and decreasing order conditions $(M=8.5$ trials, $S E M=$ 0.95 vs. $M=9.1$ trials, $S E M=0.9), t(22)<1, p=.7$, independent-samples t-test). The two-way ANOVA on mean habituation looking times with order condition (increasing vs. decreasing) as the between-participants factor and habituation trials (first three vs. last three) as the withinparticipants factor confirmed the presence of a significant overall decrease from the first three to

8 the last three habituation trials, $F(1,22)=25.69, M S e=3589.8, p<.001, \eta_{p}{ }^{2}=.54$, with no main 9 effect or interaction involving the factor order condition $(p s>.7)$ (Figure 4).

To determine whether infants' looking times during test trials differed for the novel and familiar order, mean looking times at test were entered into a four-way ANOVA with order condition (increasing vs. decreasing) and test trial order (familiar first vs. novel first) as betweenparticipants factors, and test trial pair (first vs. second vs. third) and test trial type (familiar vs. novel) as within-participants factors. The analysis revealed a significant main effect of test trial $\operatorname{pair}\left(F(2,40)=9.31, M S e=234.199, p<.001, \eta_{p}{ }^{2}=.32\right)$, with a progressive decrement of looking time across pairs (first pair: $M=9.67 \mathrm{~s}, S E M=1.15$; second pair: $M=6.32 \mathrm{~s}, S E M=$ 0.62 ; third pair: $M=5.49 \mathrm{~s}, S E M=0.6$; first pair vs. second pair: $p<.01$; first pair vs. third pair: $p<.001$; second pair vs. third pair: $p=.7$, Tukey's HSD post-hoc tests). The Test trial type main effect was also significant $\left(F(1,20)=19.14, M S e=210.645, p<.001, \eta_{p}{ }^{2}=.49\right)$, with infants looking longer to the novel $(M=8.37 \mathrm{~s}, S E M=0.77)$ than to the familiar $(M=5.95 \mathrm{~s}, S E M=$ 0.53 ) test trials. No other main effect or interaction attained significance (all $F \mathrm{~s}<2.9$, all $p \mathrm{~s}>$ .1). 
2 with 10 out of 12 infants in the increasing habituation condition $(p=.04)$, and 11 out of 12

3 infants in the decreasing habituation condition $(p<.01)$ looking longer to the novel order at test.

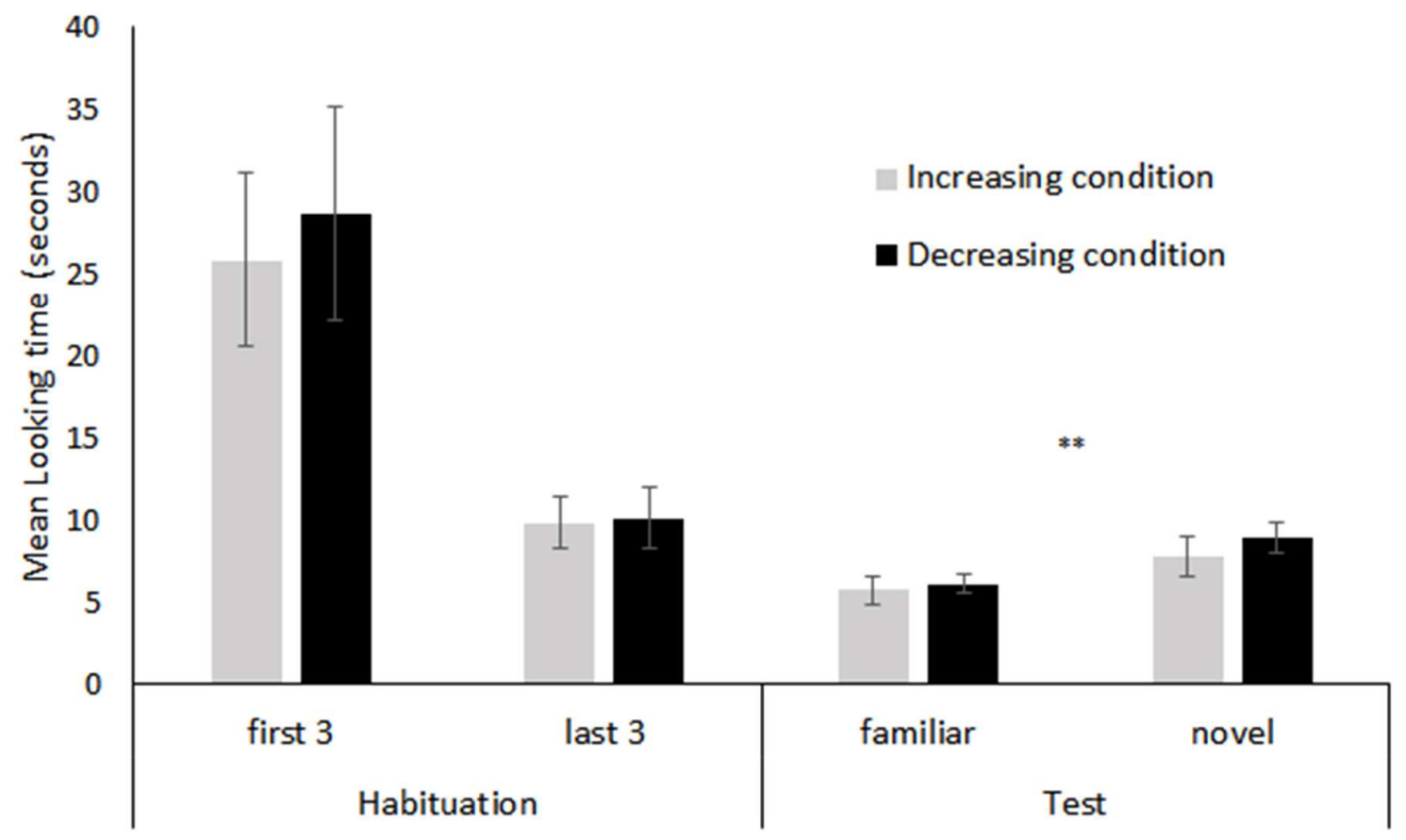

5 Figure 4. Overall mean total looking time $( \pm \mathrm{SEM})$ to the first three and last three habituation trials, and to the

6 familiar and novel test trials displayed by infants in the increasing and decreasing order conditions of Experiment 2.

7 Infants showed overall longer looking times to the novel than to the familiar sequence at test $(* * p<.01)$.

8

Results show that infants succeeded at detecting the reversal in ordinal information for temporal sequences in Experiment 2, where the durations of the stimuli were shortened to facilitate processing. In Experiment 3, we aimed at replicating this findings in a new group of 4month-olds, by further shortening the duration of the sequence stimuli. 


\section{Experiment 3}

In Experiment 3, a new group of 4-month-old infants was habituated with a series of

3 either increasing or decreasing temporal sequences and were then tested with new sequences

4 displaying the familiar and the novel orders in alternation. The durations of the audiovisual items

5 and the whole sequences were further shortened. The reason to shorten even more the stimuli

6 durations in Experiment 3 was twofold. On the one hand, we aimed at replicating the finding of

7 Experiment 2 with a new sample of babies and varying the stimulation in order to be able to

8 generalize from an eventual success. On the other hand, given that shortening the duration from

9 Experiment 1 to Experiment 2 changed dramatically the results, we wanted to investigate

10 whether a stronger effect could be observed by lowering the infants' cognitive load even more.

11

12

13

14

15

16

17

18

19

20

21

22

$\underline{\text { Methods }}$

The methods were the same as in Experiment 2 except as follows.

\section{Participants}

The final sample included 24 4-month-olds (14 females, mean age $=4$ months 7 days, range $=3$ months 28 days -4 months 20 days). Data from an additional 18 infants were discarded due to fussiness resulting in failure to complete all testing trials $(\mathrm{n}=12)$, looking time in at least one test trial more than 3SD from the overall group mean $(n=5)$, or parental interference $(n=1)$.

Stimuli 
The habituation and test sets were identical to those used in Experiments 1 and 2 but

2 differed for the duration of presentation of the geometrical figures as well as the associated tones.

3 In particular, the first stimulus set, made of green triangles, had a duration of $0.25,0.5,1 \mathrm{~s}$ for the

4 increasing habituation condition, and 1, 0.5, $0.25 \mathrm{~s}$ for the decreasing habituation condition; the

5 red squares had a duration of $0.35,0.7,1.4 \mathrm{~s}$ for the increasing habituation condition, and 1.4,

$60.7,0.35 \mathrm{~s}$ for the decreasing habituation condition; the blue circles had a duration of $0.45,0.9$,

$7 \quad 1.8 \mathrm{~s}$ for the increasing habituation condition, and 1.8, $0.9,0.45 \mathrm{~s}$ for the decreasing habituation

8 condition. The set of stimuli presented during test, made of purple rectangles, had a duration of

$90.3,0.6,1.2 \mathrm{~s}$ for the increasing sequence and $1.2,0.6,0.3 \mathrm{~s}$ for the decreasing sequence. Intercoder agreement, as computed on total fixation times on each of the six test trials

11 was $r=.956$ (Pearson correlation).

$\underline{\text { Results and Discussion }}$

In both the increasing and decreasing order conditions infants required an equivalent

number of trials to habituate $(M=8.1$ trials, $S E M=0.92$ vs. $M=7.3$ trials s, $S E M=0.71), t(22)$

$<1, p=.53)$. The two-way ANOVA on mean habituation looking times with order condition (increasing vs. decreasing) as the between-participants factor and habituation trials (first three vs. last three) as the within-participants factor confirmed the presence of a significant overall decrease from the first three $(M=31.1 \mathrm{~s}, S E M=4.8)$ to the last three habituation trials $(M=11.2$ $\mathrm{s}, S E M=1.4), F(1,22)=23.85, M S e=4754.5, p<.001, \eta_{p}{ }^{2}=.52$, with no main effect or interaction involving the factor order condition $(p s>.7)($ Figure 5).

A four-way ANOVA was performed on mean looking times during test trials to determine whether infants' performance at test revealed the detection of order reversal. The 
1 ANOVA included the between-subjects factors order condition (increasing vs. decreasing) and test trial order (familiar first vs. novel first), and the within-subjects factors test trial pair (first vs. second vs. third) and test trial type (familiar vs. novel). Results revealed a significant main effect of test trial type, $F(1,20)=13.767, M S e=1021.35, p=.001, \eta_{p}{ }^{2}=.41$, as infants looked significantly longer to the novel than to the familiar test order $(M=12.93 \mathrm{~s}, S E M=1.88$ vs. $M=$ $7.6 \mathrm{~s}, S E M=0.98$ ), irrespective of the order condition they had been habituated to (Figure 5). There was also a main effect of test trial pair, $F(2,40)=3.23, M S e=401.89, p=.05, \eta_{p}{ }^{2}=.14$, as well as a significant Test trial pair $\mathrm{x}$ Test trial type interaction, $F(2,40)=4.13, M S e=387.6$, $p=.02, \eta_{p}{ }^{2}=.17$, as the difference in looking times between familiar and novel trials was significant for the second trial pair (familiar: $7.23 \mathrm{~s}$ vs. novel: $17.27 \mathrm{~s} ; p<.01$ ), while it was not significant in the first pair (familiar: $8.13 \mathrm{~s}$ vs. novel: $15.06 \mathrm{~s} ; p=.1$ ) and was absent in third pair (familiar: 7.44 s vs. novel: $6.45 \mathrm{~s} ; p=.9$ ). Critically, the looking time to the familiar order did not change across trial pairs (first: $M=8.13 \mathrm{~s}$; second: $M=7.23 \mathrm{~s}$; third: $M=7.44 \mathrm{~s}$; all $p \mathrm{~s}=$ .99 ), while the looking time to the novel order differed between the first and third pairs (first: $M$ $=15.06 \mathrm{~s} v s$. third: $M=6.45 \mathrm{~s}, p=.03$ ), and between the second and third pairs (second: $M=$ $17.27 \mathrm{~s} v s$. third: $M=6.45 \mathrm{~s}, p=.004$ ), but was similar for the first and second pairs (first: $M=$ 15.06 s vs. second: $M=17.27 \mathrm{~s} ; p=.97$; all Tukey's HSD post hoc tests). No other main effects or interactions attained significance (all $F \mathrm{~s}<.9, p \mathrm{~s}>.36$ ).

Binomial tests confirmed a reliable effect of test trial type for both order conditions, as 10 out of 12 infants within each condition (both $p \mathrm{~s}=.04$ ) looked longer to the novel order at test. 


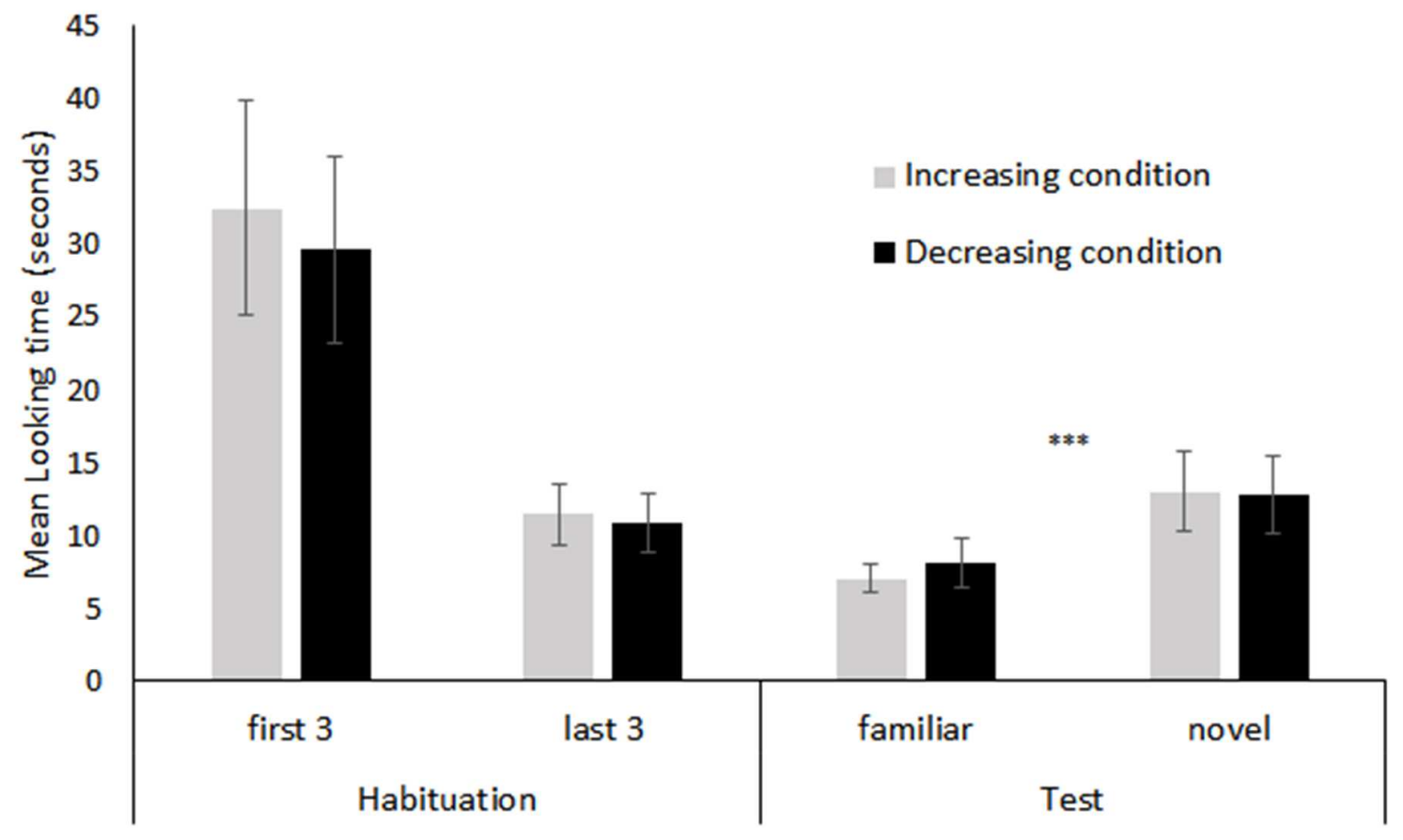

4

5 Figure 5. Overall mean total looking time $( \pm$ SEM) to the first three and last three habituation trials, and to the

6 familiar and novel test trials displayed by infants in the increasing and decreasing order conditions of Experiment 3.

7 Infants showed overall longer looking times to the novel than to the familiar sequence at test $(* * * p<.001)$.

8

9 Overall, results indicate that, in line with Experiment 2, infants in the current experiment

10 discriminated at test a reversal in ordinal direction for temporal sequences, showing reliably

11 higher looking times at test to the novel order. These findings reveal that 4-month-old infants are

12 able to compute ordinal relationships embedded in a series of duration-based sequences, and do

13 so irrespective of the order direction, increasing vs. decreasing. The absence of an asymmetric

14 performance in order discrimination, previously described for the dimensions of number and size 
at this same age, indicates that ordering operations exhibit different behavioral signatures across dimensions of magnitude.

\section{Comparisons across the three experiments}

Together, the results of the three reported experiments suggest that manipulating the temporal durations of the ordered audiovisual sequences played a critical role in modulating infants' performance, most likely by affecting the amount of cognitive load imposed to the infants by the learning task. An ANOVA with Experiment (Exp 1 vs. Exp. 2 vs. Exp. 3) as the between-subjects factor and test trial type (familiar vs. novel) and test trial pair (first vs. second vs. third) as the within-subjects factors revealed a significant main effect of test trial type, $F(1,69)=21.68, M S e=868.93, p<.001, \eta_{p}{ }^{2}=.24$, as well as of test trial pair, $F(2,138)=16.46$, $M S e=935.94, p<.001, \eta_{p}{ }^{2}=.19$ and, more crucially, a significant Experiment $\mathrm{x}$ Test trial type interaction, $F(2,69)=4.79, M S e=192.04, p=.01, \eta_{p}{ }^{2}=.12$, in the absence of a significant main effect of Experiment, $F(2,69)=2.38, M S e=352.3, p=.1, \eta_{p}{ }^{2}=.06$. Finally, there were also a significant Test trial type $\mathrm{x}$ Test trial Pair, $F(2,138)=3.51, M S e=153.13, p=.03, \eta_{p}{ }^{2}=.048$, and an Experiment $\mathrm{x}$ Test trial type $\mathrm{x}$ Test trial Pair interaction, $F(4,138)=2.94, M S e=128.45$, $p=.02, \eta_{p}{ }^{2}=.079$. This interaction was explored by Tukey's HSD post hoc tests showing that looking times to the novel order in pairs 1 and 2 of Experiment 3 were higher than looking times to the novel order of pair 3 of Experiment 1 and pairs 2 and 3 of Experiment 2 (all $p s<.03$ ).

To directly test the impact of progressively decreasing the duration of the stimuli across the three experimental conditions on infants' performance, we calculated a novelty preference score by subtracting the time spent looking at the familiar test trials to the time spent looking at the novel test trials for the three experiments. A linear regression on the novelty preference 
1 scores across the three experiments yielded a significant linear increase in the looking time to the

2 novelty at test as the cognitive load decreased from Experiment 1 to $3(r=.35, p<.01)$. One-

3 sample t-tests comparing difference scores for each experiment to zero revealed that for

4 Experiment 1 there was no significant novelty preference $(M=0.76 \mathrm{~s}, \operatorname{SEM}=1.07 ; t(23)<1, p=$

5.5 , Cohen's $d=.145)$, and that the novelty preference was reliable for both Experiment $2(M=$

$6 \quad 2.42 \mathrm{~s}, S E M=0.57 ; t(23)=4.22, p<.001$, Cohen's $d=.861)$ and Experiment $3(M=5.33 \mathrm{~s}$,

$7 \quad S E M=1.36 ; t(23)=3.91, p<.001$, Cohen's $d=.798)$ (Figure 6).

8

9

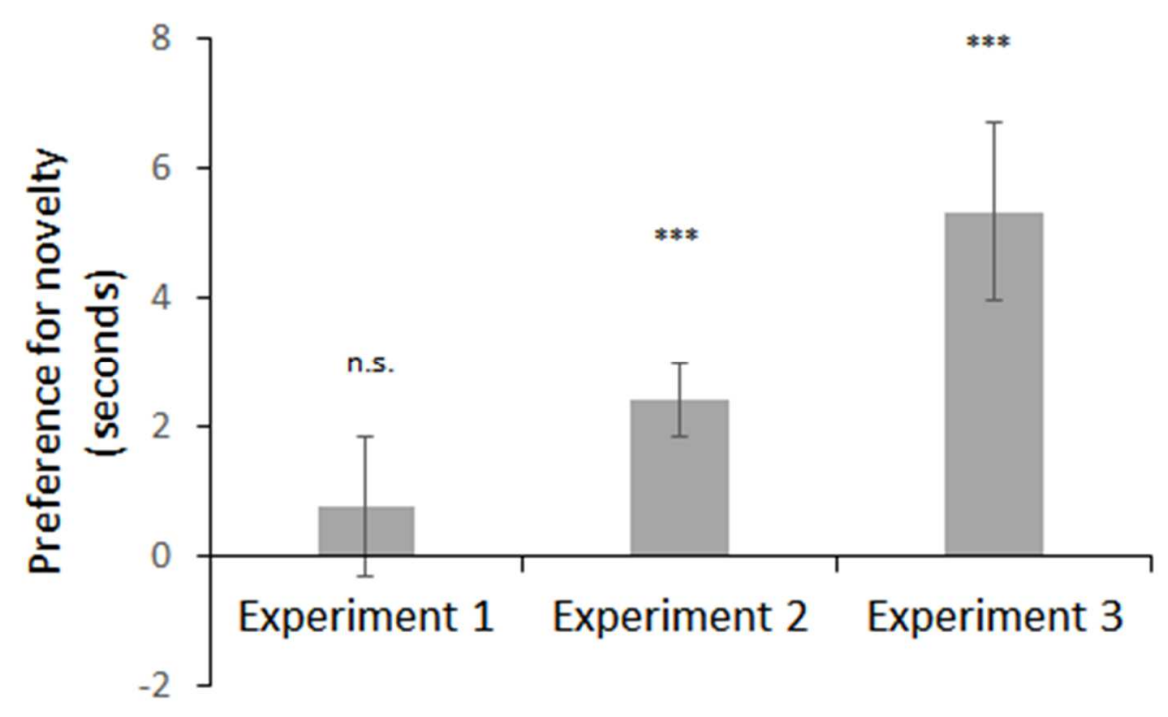

10

11 Figure 6. Overall mean $( \pm$ SEM) difference in looking time (novel-familiar) for the three experiments. The novelty 12 preference at test increased with progressively shorter durations of the stimuli across experiments (n.s. $p=.5$; *** $p$ $13<.001)$.

14

15

16

17

18 


\section{General Discussion}

This study investigated 4-month-old infants' ability to discriminate ordinal information embedded in temporal sequences. Previous research has shown that infants in the second half of their first year of life (i.e., from 7 to 12 months) are able to compute ordinal relationships for the dimensions of number, space (or size) and time (Brannon, 2002; de Hevia \& Spelke, 2010; de Hevia et al., 2017; Picozzi et al., 2010; Srinivasan \& Carey, 2010). Critically, recent studies have disclosed the presence in younger infants of an asymmetrical signature in ordinal processing for size (Macchi Cassia et al., 2012) and number (de Hevia et al., 2017), such that 4-month-olds are able to encode and represent increasing, but not decreasing, order. In light of this evidence, in the present study we aimed at further exploring younger infants' ability to process ordinal information for the domain of time, and investigate whether the same asymmetric processing signature described for number and size extends to the dimension of temporal duration.

To this aim, in three experiments we tested infants in an ordinal task in which they had to extract and represent increasing or decreasing order within duration-based audiovisual sequences and then respond to new sequences that exhibited the familiar and the novel orders in alternation. The presence of an ability to discriminate ordinal direction was inferred from infants' longer looking times to test sequences that exhibited the new order, relative to the order to which infants were habituated to. Results showed that infants were not consistently able to extract and discriminate ordinal information when the cognitive load imposed by the long temporal duration of the audiovisual sequences was high (Experiment 1), but they succeeded when the duration of the sequences was shortened (Experiments 2 and 3). Crucially, infants' discrimination performance in the presence of short sequence durations did not differ for increasing and decreasing order, indicating that infants were equally able to extract and represent ordinal 
1 information in both directions. This finding is at odds with previous demonstrations of asymmetric sensitivity to increasing versus decreasing number and size, suggesting that ordering operations exhibit different behavioral signatures across magnitude dimensions, casting doubt on the idea of a single representational system underlying these dimensions (e.g. Walsh, 2003). The results from the present study seem to be at odds with previous research showing similarities in quantitative processing among the dimensions of number, size, and time in infants

7 (de Hevia et al., 2014; de Hevia \& Spelke, 2010; Longo \& Lourenco, 2010; Srinivasan and

8 Carey, 2010). Please note, however, that previous research tested either computational processes

9 other than extracting ordinal information, or infants either younger or older than the infants tested in the present research. Crucially, in previous studies a pair of quantitative dimensions, number and time, number and space, or time and space, were presented to infants either simultaneously (as in de Hevia et al., 2014; Srinivasan \& Carey, 2010) or sequentially (as in de Hevia \& Spelke, 2010; Lourenco \& Longo, 2010). Presenting infants with two quantitative dimensions might have prompted them to align one dimension to the other, resulting in a performance pattern that plausibly reflects similarities, as opposed to dissimilarities, in the processing of those dimensions. For instance, newborn infants show evidence of expecting that a (single) increase/decrease of numerical and/or temporal information is paired with a corresponding increase/decrease in spatial extent (de Hevia et al., 2014). In contrast, asking infants to encode the ordinal relations within one single quantitative dimension (either number, size, or time), as was done both in past (de Hevia et al., 2017 and Macchi Cassia et al., 2012) and in the present research, might have favored the observation of dissimilarities in processing across dimensions. 
Much work in the past has been devoted to highlight the similarities in the processing of

2 quantitative dimensions of number, space and time, providing strong evidence that these

3 dimensions share functional similarities, are spontaneously mapped onto one another, and are

4 mentally organized along a spatial continuum, in adults (e.g., Bonato, Zorzi, \& Umilta, 2012;

5 Bueti \& Walsh, 2009; de Hevia, 2016b; de Hevia, Vallar, \& Girelli, 2008; Ren, Nicholls, Ma, \&

6 Chen, 2011; Sellaro, Treccani, Job, \& Cubelli, 2015), children (e.g., de Hevia, Vanderslice, \&

7 Spelke, 2012; de Hevia \& Spelke, 2009), infants (e.g., Bulf et al., 2016; de Hevia, 2016a;

8 Feigenson, 2007), and non-human animals (e.g., Rugani \& de Hevia, 2017).

9 However, recent work is also disclosing the existence of differential cognitive attributes

10 characterizing the processing of different magnitudes across the lifespan. For instance, at the

11 behavioral level, associations between number and spatial positions have been described at birth and in the first year of life, but these do not apply to the dimensions of size and time (Bulf et al., 2016; de Hevia et al., 2017). Moreover, different developmental trajectories characterize the precision with which we represent number and space starting from childhood (Odic, 2018), area acuity is consistently higher than number acuity both in children and adults (Odic, Libertus, Feigenson, \& Halberda, 2013), and number and time uniquely and independently correlate with school math abilities (Odic et al., 2016). It has also been shown that, in adults, differential contextual effects modulate the processing of number and time within the same individuals (Young \& Cordes, 2013). Finally, at the neural level, distinctive topographical maps and different representational codes have been disclosed for the dimensions of size and number in 21 common areas of the parietal cortex (Borghesani, de Hevia, Viarouge, Eger, \& Piazza, 2019; Harvey, Fracasso, Petridou, \& Dumoulin, 2015). The current results add to this body of 
1 literature, offering evidence that different constraints affect the processing of ordinal information

2 for the dimensions of time, as compared to number and size.

Why size and number, but not duration, show the asymmetry signature in sensitivity to

4 ordinal information early in infancy? The asymmetric ordinal processing disclosed for the

5 dimensions of number and size has been tentatively interpreted as resulting from our

6 evolutionary history (de Hevia et al., 2017). In particular, the authors invoke the developmental

7 role of the early avoidance reaction to looming stimuli - i.e., objects perceived as progressively

8 approaching the observer -, which has been observed in both humans and non-human animals

9 from birth (Ball \& Tronick, 1971; Náñez, 1988; Orioli, Bremner, \& Farroni, 2018). Unlike

10 zooming (contracting) stimuli -i.e., objects perceived as receding from the observer's location-,

11 looming stimuli have alerting effects as they enhance detection and reaction towards increasing stimuli in both infants (Walker-Andrews \& Lennon, 1985) and adults (Cléry, Guipponi,

Odouard, Wardak, \& Ben Hamed, 2015), which would produce a facilitation in the processing of the ordinal relations entailed by looming stimuli. In the domain of time, however, or at least in the way we manipulated this dimension in the current study, this perceptual phenomenon does not necessarily take place, as an increase vs. a decrease in duration entails no optical or visual changes in the object(s) considered. From this perspective, our duration-based sequences did not tap onto this primitive mechanism, and this may well account for the absence of an asymmetry signature in ordinal processing, i.e., processing advantage for increasing relative to decreasing order.

However, it should be noted that in previous studies using object size and numerosity as stimuli, objective looming effects were also absent, in the case of size through methodological controls designed to neutralize this optical effect (Macchi Cassia et al., 2012), and in the case of 
numerosity due to the variability in size and position of the elements (de Hevia et al, 2017). It was therefore suggested that, as a result of the alerting effect associated to perceptual looming, which entails increasing order, infants might develop a general (abstract) processing advantage for the ordinal information embedded in increasing sequences. This would explain why, even in the absence of objective looming stimuli, processing of increasing magnitude is enhanced and/or privileged in 4-month-old infants. However, this general account should be put further to test, as it does not seem to apply to the temporal duration stimuli used in the present study. A different, ecological account, known as 'Embodied Arithmetic' (Lakoff \& Nuñez, 2000), interprets the ascending order advantage as emerging from extensive experience with natural objects that grow in size across time, such that associations of the type 'early in time - small size' and 'later in time - large size' are created. However, the fact that temporal duration is an abstract entity with no physical substrate, as opposed to the dimensions of number and size, and that it is non dissociable from the passing of time itself, might also explain the absence of the asymmetry phenomenon for this dimension under the 'Embodied Arithmetic' account.

A second important finding of this study was that the magnitude of the novelty preference (looking time to novel - looking time to familiar) was modulated by the duration of the temporal stimuli used, such that looking time to novel test trials increased as the duration of the stimuli decreased across the three experiments. One factor that might have played a role in producing this effect is the use of suprasecond $(>1 \mathrm{~s})$ and subsecond $(<1 \mathrm{~s})$ durations. Studies with human and non-human animals have provided evidence that these two timing ranges are regulated via two distinct cognitive and neural systems (e.g., Breukelaar \& Dalrymple-Alford, 1999; Buhusi \& Cordes, 2011; Buhusi \& Meck, 2005; Cordes \& Meck, 2014; Ivry \& Keele, 1989). Stimuli in the present experiments contained either only suprasecond durations (Experiment 1), a mixture of 
suprasecond and subsecond durations (Experiment 2), or almost exclusively subsecond durations (Experiment 3). However, since the present study was not designed to provide evidence for two distinct timing mechanisms in human infants, and both ranges were used in Experiments 2 and 3, no strong claims can be made regarding this factor. Future research could directly investigate the existence of two cognitive mechanisms supporting these two duration ranges. ordinal processing reported by previous studies (de Hevia et al., 2017 and Macchi Cassia et al., 2012), our data provide some support to one of the arguments that has been proposed to account for this phenomenon, i.e., the cognitive load. Indeed, in the current study the shorter or larger length of the ordinal sequences to be computed had an impact on infants' performance. Previous studies discussed the possibility that the asymmetry phenomenon was due to differences in the variance associated to the quantitative representations associated to ascending vs. descending order, and not necessarily to an intrinsic difficulty in computing magnitude variations along the two ordinal directions. This argument was in fact entertained in relation to the 'addition advantage' observed in adults and children: for a given quantity (e.g., 5), the representation is fuzzier when it is the result of a subtraction relative to an addition, as variance associated to each operand is added up (e.g., 10-5 vs. $3+2$ ) (Barth, La Mont, Lipton, Dehaene, Kanwisher, \& Spelke, 2006). However, we argued that fuzziness might account only partially for the asymmetry phenomenon, as when the variance of the numerical sets is controlled for, performing addition seems to be as easy as performing a simple comparison, but performance in subtraction is still worse than in a simple comparison (Gilmore \& Spelke, 2008). Moreover, the variance associated to the different quantitative displays in previous, as well as in the present study, was exactly the same for both orderings, since both increasing and decreasing orders contained the 
1 same quantities, the only difference being the direction of change. Finally, the fact that

2 decreasing order is not computed differently from a random order (where the magnitude of the

3 sequence items varies non monotonically; see Macchi Cassia et al., 2012, Exp. 1b), also speaks

4 against this interpretation (for more discussion, see de Hevia et al., 2017). Finally, it is worth

5 noting that the similarities for numerical and size ordering, together with the differences between

6 these dimensions and temporal ordering, might partially reside in the fact that while numerical

7 and size-based ordered sequences can be presented both in a simultaneous (i.e., all items

8 presented at once, for instance an array of 12) and in a sequential manner (i.e., one item after the

9 other, with one individual appearing at a time until an array of 12 is completed), time is

10 incremental, as each item of the sequence has its own duration, and therefore time-based

11 sequences are intrinsically sequential. Previous studies on ordinal processing for number and size have indeed presented infants with the magnitude information, number and size, in a simultaneous manner, with information carried by each item of the sequences appearing at once and lasting for a fixed, short, time. This is not possible when manipulating the duration of the stimuli, as in the present experiment, resulting in a major methodological difference. Indeed, in the current experiment the incremental nature of the stimuli had a major role in affecting infants' performance, with infants succeeding in the order discrimination task only when sequence durations were shortened. This further highlights the impact that the sequential nature of duration-based sequences has on the ability to process order. In fact, research on children and adults has revealed that acuity for number and size is better than for duration when numerical and size stimulation is presented simultaneously, but these differences in processing disappear when stimuli are presented sequentially (Droit-Volet, Clément, \& Fayol, 2008; see also Dormal \& Pesenti, 2013; Droit-Volet, 2010). Along these lines, a goal for future research could be to 
establish whether differences in ordinal processing between number and size on the one hand, and time on the other hand, can be accounted for by the very nature of stimuli presentation, simultaneous vs. sequential, for example by presenting 4-month-old infants with size and/or numerical sequences in which, as in the case of temporal items, magnitude information carried by each numerical/size item accumulates over time, so that some time needs to elapse before each item's value reaches its peak.

\section{Acknowledgments}

We are indebted to the families that participated in these studies. Carlo Toneatto made the program to run the experiments. We thank Lionel Cohen, Alice Debost, Sarah Hausberger and Lucile Jarielle for their help in the recruiting of participants and coding of the data. We would like to thank the reviewers, whose comments contributed to improve the paper. This research was supported by a Fyssen Foundation Research Grant and an ANR (Agence National de la Recherche Scientifique) grant (ANR-15- CE28-0003-01 NUMSPA) to MDdH.

References

Ball, W., Tronick, E., 1971. Infant responses to impending collision: Optical and real. Science, $171,818-820$.

Barth, H., La Mont, K., Lipton, J., Dehaene, S., Kanwisher, N., Spelke, E.S. (2006).

Non-symbolic arithmetic in adults and young children. Cognition, 98, 199-222.

Bonn, C. D., \& Cantlon, J. F. (2012). The origins and structure of quantitative concepts.

Cognitive Neuropsychology, 29(1-2), 149-173. 
1 Borghesani, V., de Hevia, M. D., Viarouge, A., Pinheiro-Chagas, P., Eger, E., \& Piazza, M. (2019). Processing number and length in the parietal cortex: Sharing resources, not a common code. Cortex, 114, 17-27.

Bonato, M., Zorzi, M., \& Umiltà, C. (2012). When time is space: evidence for a mental time line. Neuroscience \& Biobehavioral Reviews, 36(10), 2257-2273.

Brannon, E. M. (2002). The development of ordinal numerical knowledge in infancy. Cognition, $83,223-240$.

Brannon, E. M., Lutz, D., \& Cordes, S. (2006). The development of area discrimination and its implications for number representation in infancy. Developmental Science, 9(6), F59-F64.

Brannon, E. M., Suanda, S., \& Libertus, K. (2007). Temporal discrimination increases in precision over development and parallels the development of numerosity discrimination. Developmental Science, 10(6), 770-777.

Breukelaar, J. W., \& Dalrymple-Alford, J. C. (1999). Effects of lesions to the cerebellar vermis and hemispheres on timing and counting in rats. Behavioral neuroscience, 113(1), 78.

Bueti, D., \& Walsh, V. (2009). The parietal cortex and the representation of time, space, number and other magnitudes. Philosophical Transactions of the Royal Society of London B: Biological Sciences, 364(1525), 1831-1840.

Buhusi, C. V., \& Cordes, S. (2011). Time and number: the privileged status of small values in the brain. Frontiers in Integrative Neuroscience, 5, 67.

Buhusi, C. V., \& Meck, W. H. (2005). What makes us tick? Functional and neural mechanisms of interval timing. Nature Reviews Neuroscience, 6(10), 755. 
1 Bulf, H., de Hevia, M. D., \& Macchi Cassia, V. (2016). Small on the left, large on the right: Numbers orient visual attention onto space in preverbal infants. Developmental Science, 19(3), 394-401.

4 Cléry, J., Guipponi, O., Odouard, S., Wardak, C., \& Hamed, S. B. (2015). Impact prediction by looming visual stimuli enhances tactile detection. Journal of Neuroscience, 35(10), 41794189.

Cordes, S., \& Meck, W. H. (2014). Ordinal judgments in the rat: An understanding of longer and shorter for suprasecond, but not subsecond, durations. Journal of Experimental Psychology: General, 143(2), 710.

de Hevia, M. D. (2016a). Core mathematical abilities in infants: Number and much more. In Progress in Brain Research (Vol. 227, pp. 53-74). Elsevier.

de Hevia, M. D. (2016b). Link between numbers and spatial extent from birth to adulthood. In Continuous Issues in Numerical Cognition (pp. 37-58). Academic Press.

de Hevia, M. D., Addabbo, M., Nava, E., Croci, E., Girelli, L., \& Cassia, V. M. (2017). Infants' detection of increasing numerical order comes before detection of decreasing number. Cognition, 158, 177-188.

de Hevia, M. D., Izard, V., Coubart, A., Spelke, E. S., \& Streri, A. (2014). Representations of space, time, and number in neonates. Proceedings of the National Academy of Sciences, 201323628.

de Hevia, M. D., Lee, Y. N., \& Streri, A. (2017). The temporal dimensions in the first year of life. Timing \& Time Perception, 5(3-4), 280-296.

de Hevia, M. D., \& Spelke, E. S. (2010). Number-space mapping in human infants. Psychological Science, 21(5), 653-660. 
1 de Hevia, M. D., Veggiotti, L., Streri, A., \& Bonn, C. D. (2017). At birth, humans associate "few" with left and "many" with right. Current Biology, 27(24), 3879-3884.

3 Dormal, V., \& Pesenti, M. (2013). Processing numerosity, length and duration in a threedimensional Stroop-like task: towards a gradient of processing automaticity? Psychological research, 77(2), 116-127.

Droit-Volet, S. (2010). Speeding up a master clock common to time, number and length? Behavioural processes, 85(2), 126-134.

Droit-Volet, S., Clément, A., \& Fayol, M. (2008). Time, number and length: Similarities and differences in discrimination in adults and children. The Quarterly journal of experimental psychology, 61(12), 1827-1846.

Feigenson, L. (2007). The equality of quantity. Trends in Cognitive Sciences, 11(5), 185-187.

Gallistel, C., \& Gelman, R. (2000). Non-verbal numerical cognition: From reals to integers. Trends in Cognitive Sciences, 4, 59-65

Gilmore, C. K., \& Spelke, E. S. (2008). Children's understanding of the relationship between addition and subtraction. Cognition, 107(3), 932-945.

Hamamouche, K., Keefe, M., Jordan, K. E., \& Cordes, S. (2018). Cognitive Load Affects Numerical and Temporal Judgments in Distinct Ways. Frontiers in Psychology, 9.

Harvey, B. M., Fracasso, A., Petridou, N., \& Dumoulin, S. O. (2015). Topographic representations of object size and relationships with numerosity reveal generalized quantity processing in human parietal cortex. Proceedings of the National Academy of Sciences, 112(44), 13525-13530.

Ivry, R. B., \& Keele, S. W. (1989). Timing functions of the cerebellum. Journal of Cognitive Neuroscience, 1(2), 136-152. 
1 Johnson, D. M. (1939). Confidence and speed in the two-category judgment. Archives of Psychology, 241, 1-52.

3 Lakoff, G., \& Núñez, R. E. (2000). Where mathematics comes from: How the embodied mind brings mathematics into being. Basic Books.

5 Lipton, J. S., \& Spelke, E. S. (2003). Origins of number sense: Large-number discrimination in human infants. Psychological Science, 14(5), 396-401.

Macchi Cassia, V. M., Picozzi, M., Girelli, L., \& de Hevia, M. D. (2012). Increasing magnitude counts more: Asymmetrical processing of ordinality in 4-month-old infants. Cognition, 124, 183-193.

Meck, W. H. (Ed.). (2003). Functional and neural mechanisms of interval timing. CRC Press.

Meck, W. H., \& Church, R. M. (1983). A mode control model of counting and timing processes. Journal of Experimental Psychology: Animal Behavior Processes, 9(3), 320.

Náñez Sr, J. (1988). Perception of impending collision in 3-to 6-week-old human infants. Infant Behavior and Development, 11(4), 447-463.

Odic, D. (2018). Children's intuitive sense of number develops independently of their perception of area, density, length, and time. Developmental Science, 21(2), e12533.

Odic, D., Libertus, M. E., Feigenson, L., \& Halberda, J. (2013). Developmental change in the acuity of approximate number and area representations. Developmental Psychology, 49(6), 1103.

Odic, D., Lisboa, J. V., Eisinger, R., Olivera, M. G., Maiche, A., \& Halberda, J. (2016). Approximate number and approximate time discrimination each correlate with school math abilities in young children. Acta Psychologica, 163, 17-26. 
1 Orioli, G., Bremner, A. J., \& Farroni, T. (2018). Multisensory perception of looming and receding objects in human newborns. Current Biology, 28(22), R1294-R1295.

Picozzi, M., de Hevia, M. D., Girelli, L., \& Cassia, V. M. (2010). Seven-month-olds detect ordinal numerical relationships within temporal sequences. Journal of Experimental Child Psychology, 107, 359-367.

Ren, P., Nicholls, M. E., Ma, Y. Y., \& Chen, L. (2011). Size matters: non-numerical magnitude affects the spatial coding of response. PLoS One, 6(8), e23553.

Sellaro, R., Treccani, B., Job, R., \& Cubelli, R. (2015). Spatial coding of object typical size: evidence for a SNARC-like effect. Psychological Research, 79(6), 950-962.

Srinivasan, M., \& Carey, S. (2010). The long and the short of it: On the nature and origin of functional overlap between representations of space and time. Cognition, 116(2), 217241.

Stevens, J. C., \& Marks, L. E. (1965). Cross-modality matching of brightness and loudness. Proceedings of the National Academy of Sciences, 54(2), 407-411.

VanMarle, K., \& Wynn, K. (2006). Six-month-old infants use analog magnitudes to represent duration. Developmental Science, 9(5), F41-F49.

Van Oeffelen, M. P., \& Vos, P. G. (1982). A probabilistic model for the discrimination of visual number. Perception \& Psychophysics, 32(2), 163-170.

Walker-Andrews, A. S., \& Lennon, E. M. (1985). Auditory-visual perception of changing distance by human infants. Child Development, 544-548.

Walsh, V. (2003). A theory of magnitude: common cortical metrics of time, space and quantity. Trends in Cognitive Sciences, 7(11), 483-488. 
1 Xu, F., \& Spelke, E. S. (2000). Large number discrimination in 6-month-old infants. Cognition, 2 74(1), B1-B11.

3 Young, L. N., \& Cordes, S. (2013). Fewer things, lasting longer: The effects of emotion on 4 quantity judgments. Psychological Science, 24(6), 1057-1059.

5

6

7

8 